\title{
October 2015 Pulmonary Case of the Month: I've Heard of Katy Perry
}

\author{
Kathryn E. Williams, $M B$ \\ Maxwell L. Smith, MD \\ Philip J. Lyng, MD \\ Laszlo T. Vaszar, MD
}

Department of Pulmonary Medicine

Mayo Clinic Arizona

Scottsdale, AZ

\section{History of Present IIIness}

A 45-year-old man with a history of dyslipidemia and a family history of early coronary artery disease (CAD) underwent coronary artery calcium scoring CT. He was a nonsmoker and asymptomatic.

\section{Past Medical History}

In addition to his hyperlipidemia he has a history of obesity and impaired fasting glucose.

\section{Physical Examination}

His physical examination was unremarkable.

\section{Radiography}

The thoracic $\mathrm{CT}$ was interpreted as a low risk for CAD but there were incidental findings (Figure 1).

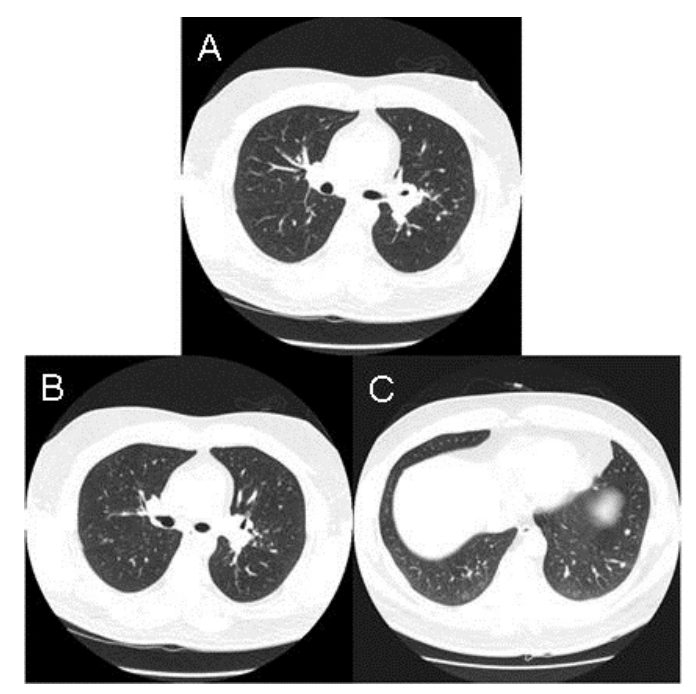

Figure 1. Representative views from the thoracic CT scan in lung windows. 
What incidental finding is not shown on the thoracic CT scan?

1. Honeycombing

2. Multiple small pulmonary nodules

3. Patchy ground glass opacities

4. Slightly enlarged mediastinal lymph nodes 


\section{Correct! \\ 1. Honeycombing}

All the above findings are present on the thoracic CT scan except honeycombing.

Honeycombing refers to clustered cystic air spaces (between 0.3-1.0 cm in diameter), which are usually subpleural and basal in distribution. It is typically a manifestation of end-stage fibrotic lung disease, often interstitial pneumonia.

What is the pattern of nodules on the thoracic CT scan?
1. Centrilobular
2. Perilymphatic
3. Random
4. Reticular 


\section{Correct! \\ 2. Perilymphatic}

Reticular refers to a pattern of too many "crisscrossing" lines creating a net-like pattern, either as a result of thickening of the interlobular septa or as a result of fibrosis as in honeycombing. Random, centrilobular and perilymphatic refer small nodular patterns on CT scan. The distribution of nodules is the most important factor, short of biopsy, for making an accurate diagnosis when small nodules are encountered at thoracic CT (1). In most cases small nodules can be placed into one of these three categories.

Random refers to no preference for a specific location in the secondary lobule (Figure 2)- nodules are seen in relation to the center of the lobule as well as the periphery of the lobule (in contact with visceral pleura and interlobular septae).

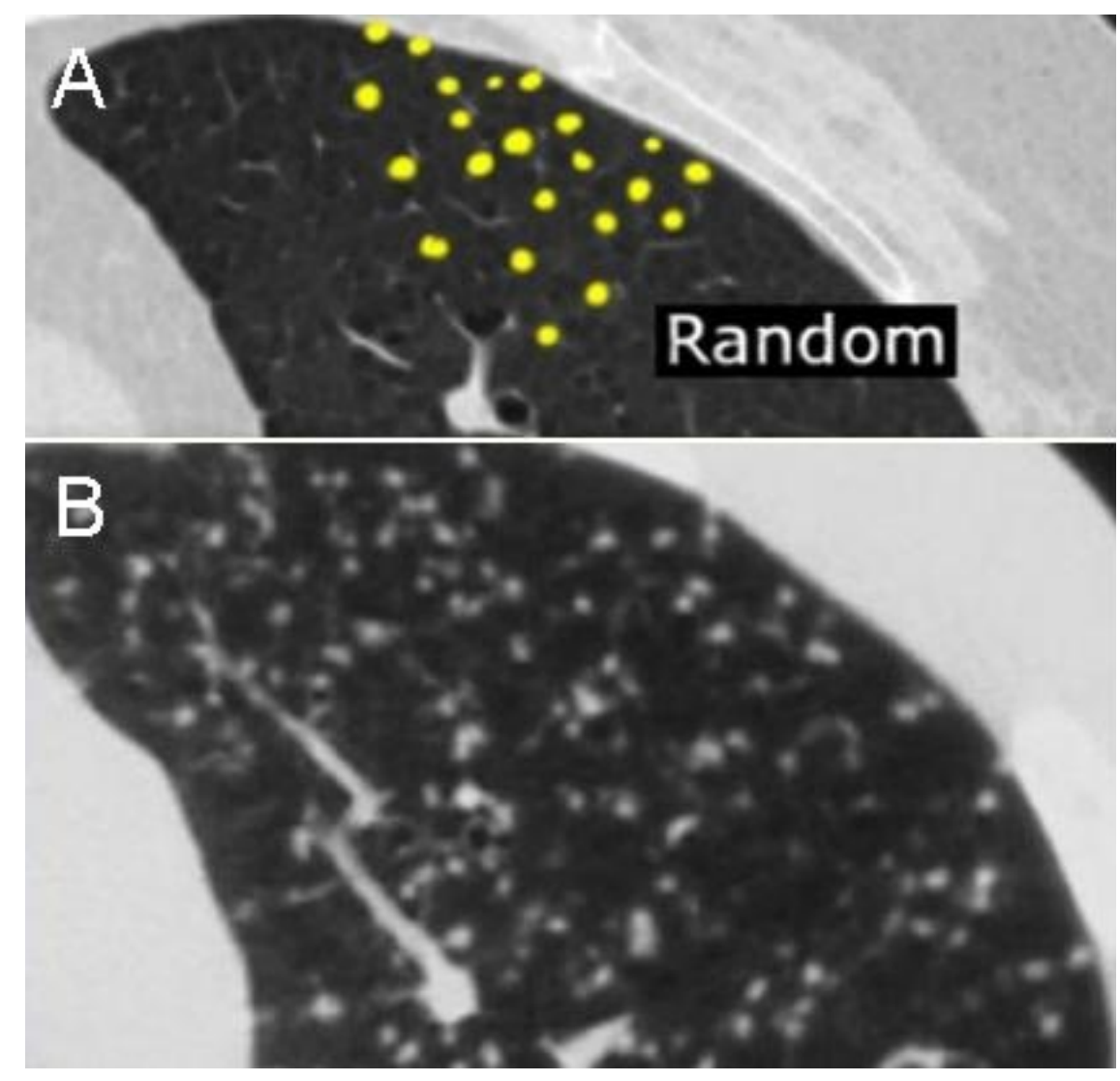

Figure 2. Panel A: Schematic showing random distribution of lung nodules. Panel B: thoracic CT scan from a patient with miliary tuberculosis showing a random pattern.

Small random nodules are seen in:

- Hematogenous metastases

- Miliary tuberculosis

- Miliary fungal infections

- Langerhans cell histiocytosis (early nodular stage) 
Unlike perilymphatic and random nodules, centrilobular nodules spare the pleural surfaces. The most peripheral nodules are centered 5-10 $\mathrm{mm}$ from fissures or the pleural surface (Figure 3).

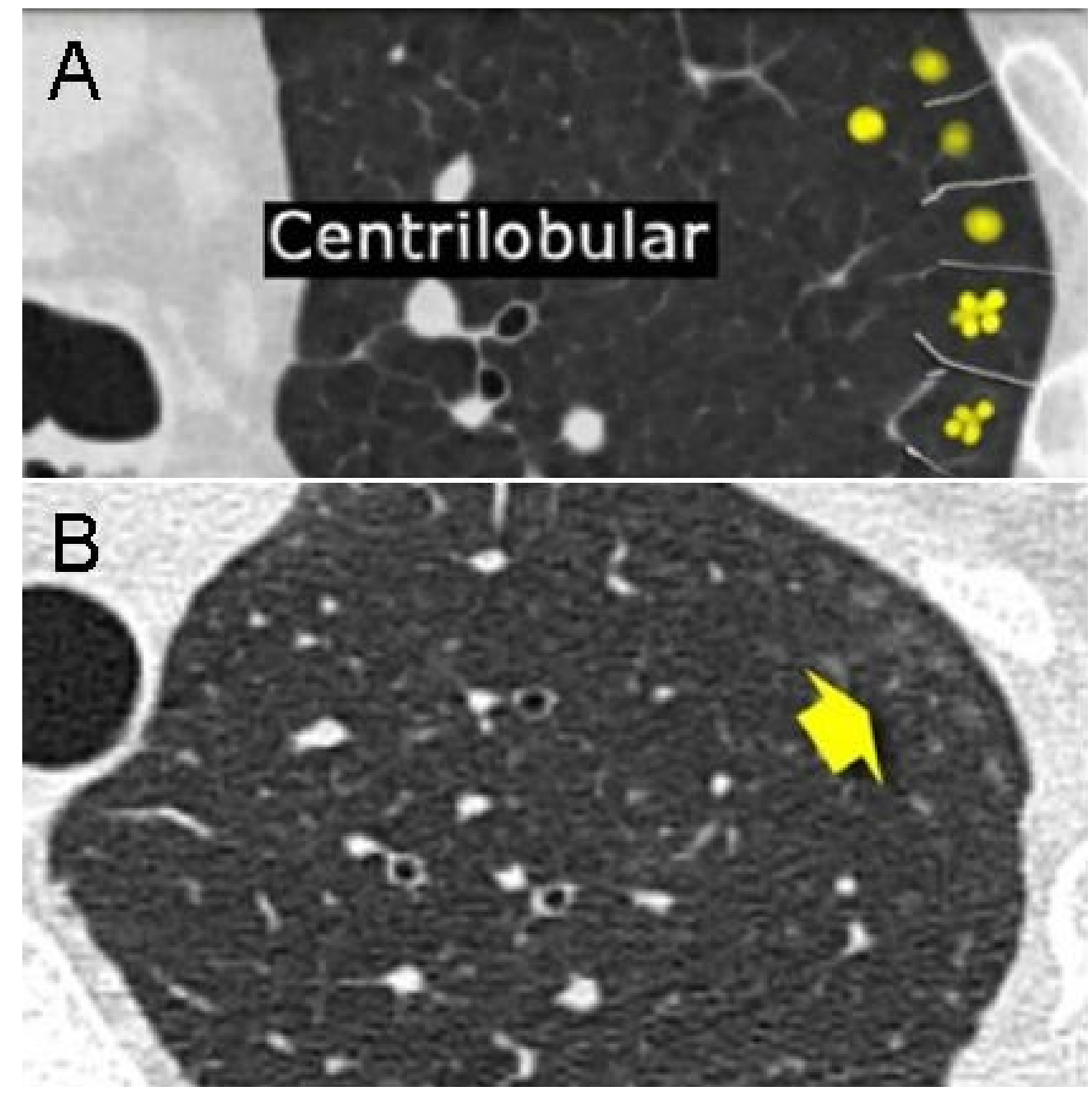

Figure 3. Panel A: schematic of centrilobular nodule distribution. Centrilobular distribution in a patient with hypersensitivity pneumonitis.

Centrilobular nodules are seen in:

- Hypersensitivity pneumonitis

- Respiratory bronchiolitis in smokers

- Some infectious airways diseases (endobronchial spread of tuberculosis or nontuberculous mycobacteria, bronchopneumonia)

In patients with a perilymphatic nodular distribution, nodules are seen in relation to pleural surfaces, interlobular septa and the peribronchovascular interstitium.

Nodules are almost always visible in a subpleural location, particularly in relation to the fissures (Figure 4). 


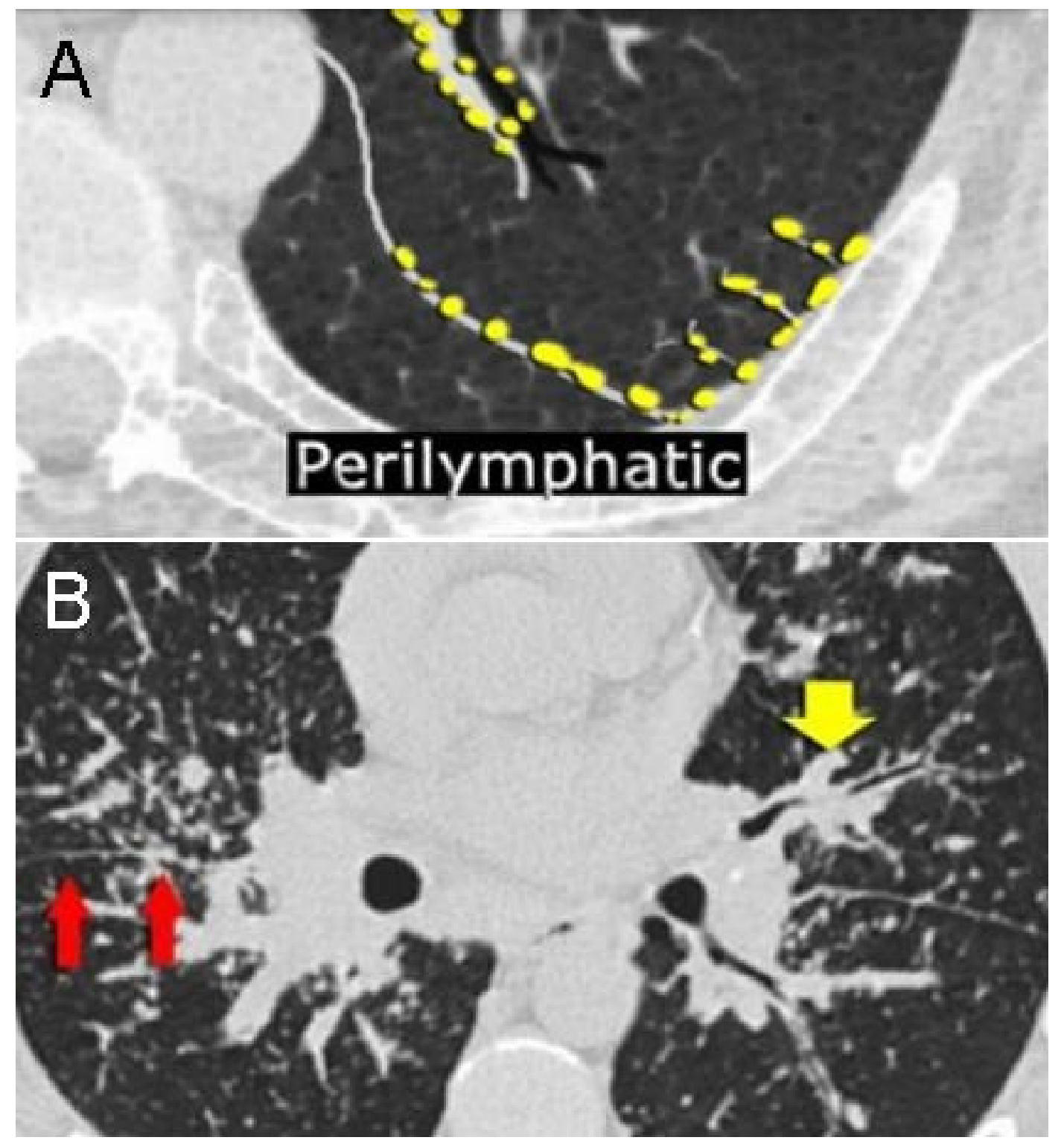

Figure 4. Panel A: schematic of perilymphatic distribution of nodules. Panel B: On the left a typical case of perilymphatic distribution of nodules in a patient with sarcoidosis. Notice the nodules along the fissures indicating a perilymphatic distribution (red arrows). In this case we see the majority of nodules located along the bronchovascular bundle (yellow arrow).

Which of the following diseases is the most common cause of a perilymphatic distribution?

1. Coal-worker's pneumoconiosis

2. Lymphangitic spread of carcinoma

3. Sarcoidosis

4. Silicosis 


\section{Correct! \\ 3. Sarcoidosis}

All are causes of a perilymphatic distribution of nodules. However, sarcoidosis would be the most common cause. In our asymptomatic patient this cause of a perilymphatic pattern would be the most likely diagnosis.

What is the next step in the diagnostic work-up of our patient?

1. Bronchoscopy with biopsy

2. Observation

3. Serum ACE level

4. Video-assisted thorascopic biopsy (VATS) 


\section{Correct! \\ 1. Bronchoscopy with biopsy}

None of the answers are absolutely incorrect. There is some controversy regarding how to proceed with a patient with fairly typical sarcoidosis such as our patient. Most would perform a biopsy to exclude other diseases and confirm the diagnosis.

At bronchoscopy some nodularity in the bronchial mucosa was noted and biopsied (Figure 5).

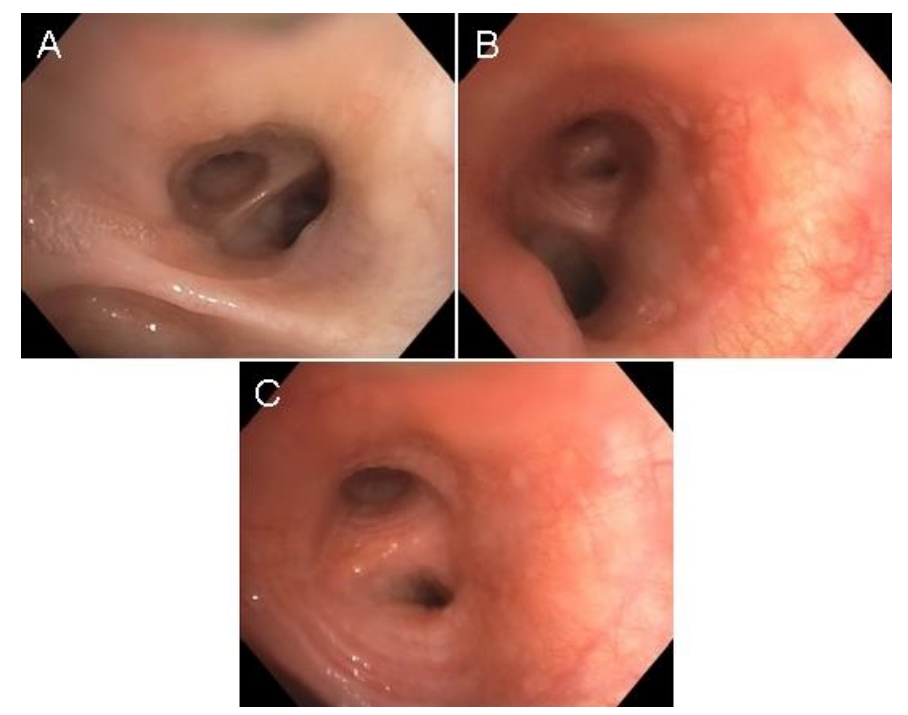

Figure 5. Views from the bronchoscopy showing bronchial nodularity.

The histology confirmed the presence of noncaseating granulomas (Figure 6).

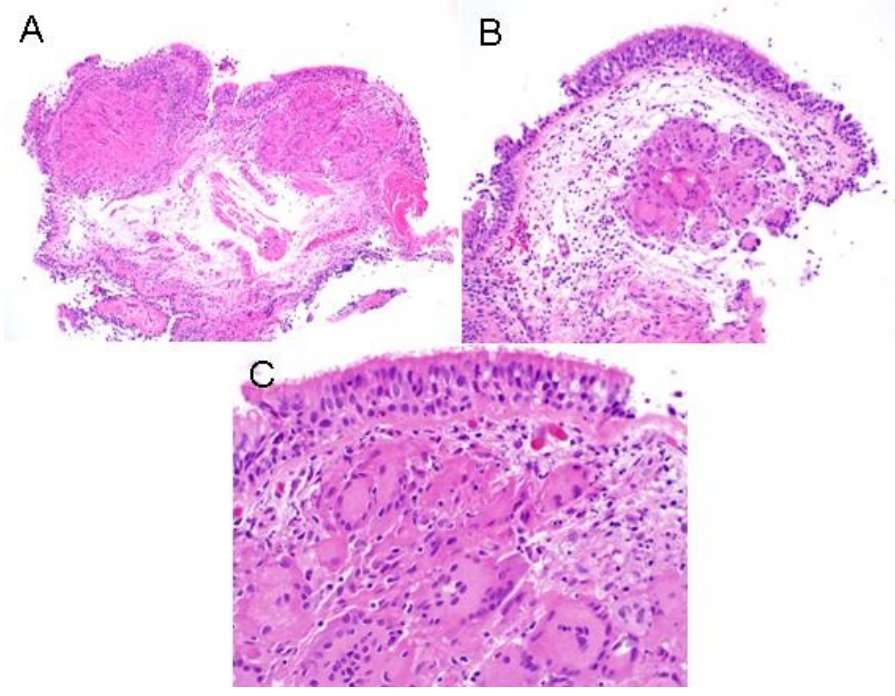

Figure 6. Low (panel A), medium (panel B) and high power (panel C) of the bronchial biopsies showing noncaseating granulomas. 
What should be done next?

1. Complete pulmonary function testing

2. Electrocardiogram

3. Eye exam

4. Serum calcium

5. All of the above 


\section{Correct! \\ 5. All of the above}

According to lannuzzi et al. (2) all should be performed. Treatment of sarcoidosis with corticosteroids is also controversial. Most treat progressive or symptomatic pulmonary disease and any extrathoracic disease with corticosteroids. However, the dosage and duration of therapy is unclear. In the case of progressive disease despite corticosteroid treatment, most treat with cytotoxic drugs such as methotrexate or cyclophosphamide. Some patients have progressive disease despite therapy and should be considered for lung transplantation.

\section{References}

1. Smithuis R, van Delden O, Schaefer-Prokop C. Lung - HRCT Basic Interpretation. Radiology Assistant. Available at:

http://www.radiologyassistant.nl/en/p42d94cd0c326b/lung-hrct-basicinterpretation.html (accessed 9/23/15).

2. Iannuzzi MC, Rybicki BA, Teirstein AS. Sarcoidosis. N Engl J Med 2007;357:215365. [CrossRef] [PubMed] 